REVESCO. Revista de Estudios Cooperativos

ISSN: $1885-8031$

http://dx.doi.org/10.5209/REVE.61636

\title{
Prácticas empresariales de innovación social en la gestión de la edad y su aplicación en el ámbito del cooperativismo ${ }^{1}$
}

\author{
Mariola Serrano Argüeso ${ }^{2}$ y Nerea Ereñaga de Jesús ${ }^{3}$
}

Recibido: 27 de septiembre de 2017 / Aceptado: 27 de noviembre de 2017

Resumen. El WP5 del Proyecto Social Innovation: Driving Force of Social Change (SI DRIVE) evidencia que gran parte de la fuerza de trabajo europea se acerca a la edad de jubilación mientras que, al mismo tiempo, la disponibilidad de mano de obra especializada para reemplazar a los jubilados es escasa. Para hacer frente a este reto algunas empresas están llevando a cabo políticas de innovación en materia de gestión de la edad para el empleo y de gestión de la edad en el empleo. El estudio trata de identificar cuáles son las medidas en el ámbito empresarial y en especial, si esa preocupación está presente en el ámbito del cooperativismo vasco.

Palabras clave: Políticas laborales; Envejecimiento activo; Empleo; Trabajadores mayores; Cooperativas.

Claves Econlit: J14; P13; K31; J21.

[en] Business practices of social innovation in age management and its application to cooperativism

Abstract. The WP5 of the Social Innovation: Driving Force of Social Change (SI DRIVE) Project shows that a large part of the European workforce is approaching the retirement age while, at the same time, the availability of qualified workers to replace these retirements is limited. Considering this challenge, some companies are implementing innovation policies in the areas of age management for employment and age management at work (on the employment). The study tries to identify which measures are currently present in the business sector and especially, if the Basque cooperative movement is concerned about this challenge.

Keywords: Labour policies; Active aging; Employment; Senior workers; Cooperatives.

Sumario. 1. Introducción. 2. Concepto y evolución del término envejecimiento activo. 3 . Envejecimiento activo y cooperativismo. 4. Innovación en materia de gestión de la edad en la empresa. 5. La gestión de la edad en el ámbito del cooperativismo en la comunidad autónoma de Euskadi. 6. Conclusiones. 7. Referencias bibliográficas.

1 Este trabajo está basado en los resultados del proyecto de investigación (Grant Agreement 612870) Social Innovation: Driving Force of Social Change (SI DRIVE), financiado por la Comisión Europea en el VII Programa Marco. www.si-drive.eu.

2 Universidad de Deusto, España

Dirección de correo electrónico: mariola.serrano@deusto.es

3 Universidad de Deusto, España

Dirección de correo electrónico: nerea.erenaga@deusto.es 
Cómo citar: Serrano Argüeso, M. y Ereñaga de Jesús, N. (2019) Prácticas empresariales de innovación social en la gestión de la edad y su aplicación en el ámbito del cooperativismo. REVESCO. Revista de Estudios Cooperativos, Primer Cuatrimestre, № 130, pp. 176-197. DOI: 10.5209/REVE.61636.

\section{Introducción}

El envejecimiento de la población es un fenómeno presente en el mundo entero con diferentes grados en cada uno de los países. Para el año 2050 casi un tercio de la población europea (el 29\%) tendrá más de 60 años (Comisión Europea, 2011: 465). Aunque el proceso de envejecimiento es generalizado en todos los países europeos en algunos, como España, lo ha sido de forma más acelerada puesto que este cambio de tendencia se ha producido en medio siglo cuando en el resto de los países comenzó hace ya más de un siglo. Además, en España, nos estamos enfrentando a un proceso de "envejecimiento del envejecimiento", que consiste en un incremento de la población mayor entre los mayores. Ante esta situación de descenso de la población en edad de trabajar y el impacto que ello puede tener en el mercado laboral, sistema sanitario y calidad de vida, desde Europa se recomienda que se pongan en marcha reformas que garanticen la viabilidad del sistema de pensiones a través de la continuidad en el mercado de trabajo de las personas mayores y el fomento de las políticas de envejecimiento activo. Uno y otro aspecto están inevitablemente unidos pero ¿cómo se están entendiendo en el ámbito empresarial? ¿Existen realmente medidas de gestión de la edad en el ámbito empresarial?

El objetivo de este estudio es identificar si existe una verdadera preocupación empresarial por implementar políticas innovadoras en materia de gestión de la edad, en especial, en el ámbito del cooperativismo vasco. A tal efecto, tras un análisis conceptual del término envejecimiento activo, se reflexionará sobre cómo el modelo cooperativo que trabaja por y para las personas debe tener presente el envejecimiento de la población y adoptar políticas empresariales para afrontar esta nueva situación. Identificaremos, entonces, cuáles son las medidas empresariales de gestión de la edad en el empleo y para el empleo y se expondrán los resultados de la encuesta realizada a un grupo de cooperativas en la Comunidad Autónoma de Euskadi. Los resultados y la necesidad de priorizar estas acciones en el ámbito empresarial serán expuestas en el apartado de conclusiones.

\section{Concepto y evolución del término envejecimiento activo}

Situamos el origen del término envejecimiento activo en EEUU, en la década de los años sesenta del siglo pasado, coincidiendo con la promulgación de la Age Discrimination Employment Act 1967, introducido por la teoría sobre "successful ageing", a la que, en 1980, se la redefine como "productive ageing" (SánchezUrán Azaña, 2011).

La Organización Mundial de la Salud (OMS) define el envejecimiento activo como "el proceso de optimización de las oportunidades de salud, participación y seguridad con el fin de mejorar la calidad de vida a medida que las personas 
envejecen". Este concepto puede aplicarse tanto a los individuos en particular como a grupos de población indistintamente, y permite a las personas realizar su potencial de bienestar físico, social y mental a lo largo de todo su ciclo vital a la vez que logran continuar participando en la sociedad de acuerdo con sus capacidades, mientras que se les proporciona una serie de cuidados y de protección adecuados a su situación personal. La OMS adoptó el concepto de "envejecimiento activo" en la década de los noventa con la intención de renovar la tradicional referencia al "envejecimiento saludable" que, en cierto modo, restringía el mensaje que se pretendía transmitir dado que no abarcaba el amplio número de características que comprende el término "envejecimiento activo"4. El envejecimiento activo es el resultado del reconocimiento de los derechos de las personas mayores y la aplicación de los principios de independencia, participación, dignidad y asistencia (Alvarado García y Salazar Maya, 2014: 61) ${ }^{5}$ y sustituye, de esta manera, el tradicional planteamiento basado en las necesidades (que contempla a las personas mayores como objetivos pasivos) por otro basado en los derechos, al reconocer los derechos de las personas mayores a la igualdad de oportunidades y de trato en todos los aspectos de la vida a medida que envejecen (Giro Miranda, 2006: 27). Así pues, "el envejecimiento activo implica entender esta etapa de la vida como un ciclo más del crecimiento personal añadiendo vida a los años y no solamente años a la vida". Pero no sólo debemos cuidar a las personas mayores por razones económicas o de salud, sino que la sociedad tiene también un claro interés en aprovechar su trabajo y experiencia.

La Comisión Europea pone en alza la importancia del envejecimiento activo como elemento clave para el porvenir y resalta la necesidad que tiene Europa de incrementar y utilizar su potencial laboral con el fin de hacer frente a la competencia mundial ${ }^{7}$. En 1999 la UE, en la Comunicación de la Comisión Europea "Hacia una Europa para todas las edades: promocionar la prosperidad y la solidaridad intergeneracional de 1999" se refiere por primera vez al término envejecimiento activo ${ }^{8}$. A esta primera etapa se la ha llamado "Etapa de recepción" y en ella se asientan las bases para configurar la futura estrategia comunitaria a las implicaciones del envejecimiento activo en el empleo, la protección social, la salud y los servicios sociales (Sánchez-Urán Azaña, 2011). La preocupación por el aumento de "trabajadores mayores" está también presente en la Estrategia de Lisboa $^{9}$ que recuerda que para conseguir el objetivo del envejecimiento activo es

4 En mayo de 2014, la $67^{\mathrm{a}}$ Asamblea Mundial de la Salud (Decisión WHA67(13)(2014) solicitó la elaboración de una estrategia y un plan de acción mundiales integrales sobre el envejecimiento y la salud. El punto de partida fue el Informe mundial sobre el envejecimiento y la salud, publicado en 2015 (http://apps.who.int). En la $69^{\text {a }}$ Asamblea Mundial de la Salud, el 22 de abril de 2016 (A69/17) se aprueba la Acción multisectorial para un envejecimiento saludable basado en el ciclo de vida: proyecto de estrategia y plan de acción mundiales sobre el envejecimiento y la salud.

5 En las últimas décadas se han propuesto varios modelos de envejecimiento que intentan dar una mirada más positiva al término de envejecimiento: envejecimiento exitoso, envejecimiento saludable y envejecimiento activo.

6 Fuente de información: http://www.envejecimientoactivo2012.net/Menu29.aspx (Última visita: 19/07/2016).

7 COMISIÓN EUROPEA, Una estrategia para un crecimiento inteligente, sostenible e integrador, Europa 20, Bruselas, 3 de marzo de 2010, no publicada en el Diario Oficial.

8 Bruselas, 21/05/1999 (COM (1999) 221. Final.

9 Que tenía como objetivo conseguir que en el año 2010 la UE fuese la economía más competitiva y dinámica del mundo. Consejo Europeo de Lisboa 23 y 24 de marzo de 2000. 
necesario que las políticas de empleo de los distintos países se centren en tres ámbitos: pleno empleo, calidad y productividad en el trabajo, y cohesión e inclusión social. El ámbito de la productividad enlaza con la preocupación empresarial por la situación de los trabajadores mayores, pero, dadas las dificultades para medir la productividad se establecen referencias concretas que a la vez que obligan a los países, permiten evaluar adecuadamente los esfuerzos realizados. De entre estos objetivos, la preocupación por el futuro demográfico lleva a aprobar en las reuniones del Consejo de Europa de los años 2001 y 2002 (Estocolmo y Barcelona) dos objetivos que en concreto están centrados en los trabajadores mayores: una tasa de empleo de un 50\% para los trabajadores de entre 55 y 64 años y un incremento de cinco años de la edad media de jubilación. Es la llamada fase de consolidación y desarrollo de la estrategia global sobre el envejecimiento activo. La convergencia de las políticas de empleo y de las pensiones a través del envejecimiento activo en y para el empleo es a partir de ese momento, uno de los debates políticos sobre empleo en la UE junto a otros como la noción y extensión de solidaridad intergeneracional en un contexto de crisis económica (Sánchez-Urán Azaña, 2011) ${ }^{10}$.

En adelante, la Comisión Europea se esfuerza por supervisar y facilitar el aprendizaje mutuo entre Estados miembros sobre la promoción de más y mejores empleos en la UE ${ }^{11}$. La Estrategia Europa 2020 establece el empleo como uno de los objetivos prioritarios. Aun así, retirarse o quedarse en el mercado de trabajo no puede ser solo el resultado de consideraciones de tipo financiero, por lo que, además de eliminar los desincentivos a la continuación en el trabajo, deben definirse medidas específicamente dirigidas a los trabajadores mayores entre las que se proponen dificultar la jubilación anticipada, estimular el aprendizaje permanente para evitar la obsolescencia de los conocimientos, adaptar las condiciones de los puestos de trabajo a las características de los trabajadores mayores, hacer que el trabajo interese más que el retiro, mantener una buena salud o facilitar el cuidado de los mayores. Por ello llama más la atención que entre los objetivos de la Estrategia Europa $2020^{12}$ no figurase ninguno específicamente

10 Cabe citar como documentos básicos de la etapa de consolidación y desarrollo de la política de envejecimiento activo las siguientes comunicaciones de la Comisión Europea: Comunicación de la Comisión al Consejo y al Parlamento Europeo, "Promover el progreso Económico y Social en un mundo en proceso de envejecimiento" (COM-2002, 143 final); Comunicación de la Comisión. Libro Verde "Frente a los cambios demográficos, una nueva solidaridad entre generaciones" (COM (2005) 94 final-no publicada en el Diario Oficial); Comunicación de la Comisión, de 12 de octubre de 2006, "El futuro demográfico de Europa: transformar un reto en una oportunidad" (COM (2006) 571 final -no publicada en el Diario Oficial); Comunicación de la Comisión al Parlamento Europeo, al Consejo, al Comité Económico y Social Europeo y Comité de las Regiones, de 10 de mayo de 2007, "Promover la solidaridad entre las generaciones (COM (2007)244 final - no publicada en el Diario Oficial); Comunicación de la Comisión al Parlamento Europeo, al Consejo, al Comité Económico y Social Europeo y al Comité de las Regiones de 29 de abril de $2009-$ abordar los efectos del envejecimiento de la población de la UE (Informe 2009 sobre envejecimiento demográfico) (COM (2009) 180 final - no publicada en el Diario Oficial-).

11 La Estrategia Europea del Empleo establece un marco para que los países de la UE compartan experiencias, aprendizaje e ideas en materia de política de empleo, mientras que la Comisión aporta un análisis general y recomendaciones. Véase el método abierto de coordinación: http://europa.eu/legislación_sumaries/glossary/open_method_coodiantio_es.htm.

12 Una de las iniciativas europeas emblemáticas de Europa 2020 es la Agenda de nuevas cualificaciones y empleos. Destaca la importancia de mejorar las cualificaciones de los trabajadores de mayor edad, especialmente los vulnerables a la restructuración económica y políticas que respalden la transición en el 
dirigido a los trabajadores mayores. Esta cuestión pretendió resolverse luego con la fijación de objetivos y prioridades comunes para todos los países de la UE a través de un conjunto de quince indicadores de los que seis están dirigidos a capturar la situación de los mayores en el mercado de trabajo (Rey, 2012: 14). Esta es la tercera etapa del envejecimiento activo en Europa: la fase de integración y sostenibilidad del crecimiento económico y social. Coincide parte de la doctrina en denunciar, como hemos visto, que en esta etapa, el envejecimiento activo no se desatiende pero queda diluido en una Estrategia Global que abarca a otros colectivos con especiales dificultades en el empleo en el llamado mercado de trabajo inclusivo y dinámico que, más allá de la prolongación de la vida activa o mantenimiento de la actividad profesional para aumentar la ratio de empleo del colectivo de personas de edad avanzada, adopta ahora una perspectiva más constructiva: envejecimiento activo en la comunidad y envejecimiento saludable (Sánchez-Urán Azaña, 2011).

El año 2012 fue declarado año europeo del envejecimiento activo y la solidaridad intergeneracional y la UE publicó una guía ${ }^{13}$ para los agentes locales donde vienen recogidas una serie de recomendaciones y planes de fomento del envejecimiento activo. Las mismas vienen divididas en 4 apartados o "niveles". En cada uno de ellos se pretende el fomento del envejecimiento activo desde una perspectiva diferente, apuntando una serie de principios que los Estados deberán observar a la hora de diseñar sus propias políticas de envejecimiento activo. Más recientemente, los interlocutores sociales de la UE han firmado un acuerdo marco autónomo dentro del programa de trabajo para 2015-2017 dónde, tras analizar conjuntamente y en profundidad la cuestión del empleo, se ofrecen recomendaciones clave para potenciar el envejecimiento activo desde un enfoque intergeneracional ${ }^{14}$. En el año 2017 también la UE ha publicado la Recomendación 2017/761 de la Comisión, de 26 de abril de 2017, sobre el pilar europeo de derechos sociales dónde, en su artículo 10.b), incide en el derecho de las personas trabajadoras a un entorno de trabajo adaptado a sus necesidades profesionales y que les permita prolongar su participación en el mercado laboral ${ }^{15}$.

Las políticas de la UE apoyan y fomentan el proceso de envejecimiento activo y la solidaridad entre generaciones, pero son los Estados miembros a escala nacional, regional, local y en los procesos de negociación colectiva los competentes para legislar, impulsar e implementar dichas políticas. Los países europeos han adoptado distintos enfoques nacionales sobre el envejecimiento activo. Así, los estados nórdicos enfocan el envejecimiento activo hacia la formación, capacitación y aprendizaje. En los países del nordeste, incluido aún el Reino Unido, el envejecimiento activo se enfoca a la eliminación de barreras, a la participación en el mercado de trabajo, incluida la discriminación por razón de edad, y la Europa Continental, más preocupada por competir en un mercado global, centra sus

mercado laboral de las personas mayores, en particular, del desempleo a un nuevo puesto de trabajo. Especialmente interesante es el programa de "Aprendizaje Permanente".

13 Concretamente, la siguiente: COMISIÓN EUROPEA, How to promote active ageing in Europe. EU support to local and regional actors, Bruselas Ed. Oficina de Publicaciones de la Comisión Europea, 2011.

14 European social partners' autonomous framework agreement on active ageing and an inter generational approach. Firmado por Business Europe, UEAPME, CEEP y ETUC el 8 de marzo de 2017.

15 Diario Oficial de la Unión Europea. L 113/56 de 29 de abril de 2017. 
políticas en introducir flexibilidad al final de la vida laboral (Ney, 2005) ${ }^{16}$. Lo cierto es que, como ha denunciado la doctrina, cualquiera que sea el enfoque sobre el envejecimiento activo adoptado por los países necesita una Estrategia integral a medio y largo plazo con medidas dirigidas a un enfoque activo y no meramente compensador de la retirada temprana del mercado de trabajo (Sánchez-Urán Azaña, 2011).

\section{Envejecimiento activo y cooperativismo}

El envejecimiento de los trabajadores no es ajeno a las empresas de Economía Social ni, en concreto, al ámbito del cooperativismo. Y es que también en este ámbito los problemas demográficos han hecho que el número de jóvenes disminuya y sea necesaria la búsqueda de un equilibrio entre pocos jóvenes y una mayor cantidad de personas adultas, con las reiteradas consecuencias de la necesidad de inmigración, los problemas de pago de las pensiones, el retraso de la edad de jubilación (Martínez Charterina, 2009: 147) y la introducción de medidas para poder gestionar políticas sociales, de calidad de vida y de inclusión social para las personas trabajadoras mayores ${ }^{17}$. En este sentido, los valores cooperativos de responsabilidad social, democracia, formación y solidaridad son claves para generar importantes cambios sistemáticos que aporten una ampliación de oportunidades laborales y sociales a las personas trabajadoras de mayor edad, o lo que es lo mismo, enfocar el envejecimiento activo como fuente de oportunidades sociales y económicas. Todo ello requiere que el envejecimiento se observe como un proceso que no sólo debe realizarse desde ámbitos tradicionales como el sociosanitario o el de las pensiones, sino desde otros más novedosos como la formación, la cultura, los valores y las formas de participación ${ }^{18}$.

Los valores cooperativos para construir una empresa viable, con la que los socios se identifican al apreciar en ella un proyecto que garantice su empleo y vida profesional, deben tener presente los derechos de los trabajadores mayores y adoptar medidas al respecto. En efecto, las sociedades cooperativas pueden jugar un papel importante en el proceso de recolocación de los trabajadores de mayor edad para prolongar su permanencia en el mercado de trabajo, de hecho, es necesario por un lado, tener presentes las contribuciones a la comunidad realizadas desde y mediante la veteranía o experiencia, y por el otro, proteger las contribuciones de la gente de mayor edad, concretamente su participación, protección y presencia en el mundo laboral (Sánchez Boza, 2012) y romper definitivamente con la idea de que las organizaciones con personas más jovenes son más dinámicas y flexibles (Molero Zayas y Buesa Blanco, 1996). A lo largo de la historia se ha puesto de manifiesto el carácter defensivo de las cooperativas en

16 Línea que también ha sido identificada en el SI DRIVE, y es que aunque se espera que la situación cambie, en el sur de Europa la participación laboral se erige todavía sobre la flexibilidad y la reducción de costes.

Fuente de información: https:/www.si-drive.eu/wp-content/uploads/2015/12/SI-DRIVE-Policy-BriefEmployment-2015.pdf (Última consulta: 24/08/2017).

17 Fuente de información: http://www.aciamericas.coop/El-adulto-mayor-y-el (Última consulta: 12/07/2017).

18 Fuente de información: http://www.innobasque.eus/microsite/innovacion_social/proyectos/envejeciendo-enpositivo/ (Última consulta: 17/07/2017). 
épocas de crisis y cómo consiguen dar respuesta a las necesidades de creación de empleo en momentos de dificultades (Martín López, 2006). Es más, su énfasis en sus propios valores ha demostrado su versatilidad y viabilidad, características que las han hecho capaces de prosperar incluso en épocas difíciles ${ }^{19}$. Y es que la filosofía cooperativa reside en la elaboración de un proyecto que trasciende lo individual. Muestra de ello son sus principios de cooperación y compromiso con la comunidad (sexto y séptimo principio respectivamente). De ambos deducimos su obligación ética ante el envejecimiento de la población, pues les lleva a preocuparse por los intereses de la sociedad y a cooperar por medio de sus estructuras y organizaciones a fortalecer la cohesión social. En este escenario, la Alianza Cooperativa Internacional ${ }^{20}$ sitúa el punto de partida para coadyuvar en el envejecimiento activo de las personas cooperativistas en la promoción de planes de acción concretos que desarrollen intervenciones integrales, de calidad y socialmente responsables, de manera que las organizaciones continúen mostrando una significativa fidelidad a su objetivo social y vocación de origen desde tres ejes principales: la promoción de estilos de vida saludable, el desarrollo y oportunidades educativas, y la accesibilidad social ${ }^{21}$, en consonancia con los denominados principios en favor de la política de edad proclamados por la Organización de las Naciones Unidas (ONU) 22 .

Pero más allá de la declaración de valores de la identidad cooperativa, existen estudios más locales que contribuyen a dar forma a la esencia de un sistema de principios y valores cooperativos. Estos estudios señalan cómo se podría incidir en estos factores desde la sociedad y desde nuestras organizaciones. Concretamente, en la línea de actuación hacia la mejora de la calidad de vida presente y futura de toda la sociedad, se pone el énfasis en el "Envejecimiento en Positivo" en el que se establecen líneas de actuación para contribuir a la calidad de vida de las personas mayores. Entre ellas: el desarrollo de productos y servicios orientados a las personas mayores y diseñados desde sus propias necesidades y derechos; la promoción de una cultura donde el envejecimiento sea valorado, y a su vez, se reconozca la contribución de las personas de mayor edad; el diseño de políticas públicas que no abarquen de manera única la protección y el cuidado de las personas mayores, sino que se destinen en igual medida a garantizar su plena intervención y participación en la sociedad; el fomento desde el ámbito de la salud de sistemas sanitarios que se desarrollen desde una perspectiva del ciclo vital completo y adquieran líneas de trabajo orientadas a promover la salud, prevenir enfermedades y acceder de manera igualitaria a todo tipo de atención sanitaria de calidad; la creación de espacios intergeneracionales donde las personas de

19 En palabras de Ban Ki-moon, Secretario General de las Naciones Unidas. Fuente de información: http://www.un.org/es/events/coopsyear/ (última consulta: 18/09/2017).

20 En adelante, ACI.

21 Así pues, el primero de los ejes que la ACI trabaja para el envejecimiento activo es el de entender las necesidades de la persona mayor asociada a sus cooperativas y conocer el estado actual de la situación para cada entidad. El segundo eje es impulsar el ajuste y la creación de servicios que satisfagan las principales necesidades y expectativas de la persona mayor asociada a sus cooperativas miembros. Y el tercero es promover la concienciación de los miembros de la cooperativa en la importancia de trabajar para mitigar los efectos del envejecimiento sobre el desarrollo socioeconómico y en adoptar medidas urgentes para garantizar su constante integración.

22 Así como, la independencia, la participación, los cuidados, la autorealización y la dignidad. 
diferentes generaciones tengan la oportunidad de convivir, comunicarse y relacionarse, todo ello concebido como nuevos espacios de intercambio y participación; la formación y sensibilización acerca del envejecimiento de la población, ya que es la llave al cambio cultural y a la aceptación del envejecimiento y la vejez, creando un modelo que incentive y motive las aportaciones de las personas mayores (Innobasque, 2010: 12). En este ámbito, existen cooperativas creadas específicamente sobre esta óptica, como en el caso concreto de Brisa del Cantábrico Sociedad Cooperativa, la cual se erige sobre los principios de envejecimiento activo anteriormente $\operatorname{citados}^{23}$.

Pues bien, es desde estos principios desde dónde el cooperativismo puede contribuir a la igualdad de oportunidades de las personas mayores y a la construcción colectiva de una estrategia de envejecimiento activo que permita llegar a una vejez dinámica y con calidad de vida ${ }^{24}$. Para cubrir las necesidades sociales hacia la búsqueda de mejorar las condiciones de bienestar a través del mercado de trabajo, el cooperativismo debe plantear y poner en práctica acciones cooperativas en torno al envejecimiento sostenible, saludable, autónomo y productivo.

Ante este escenario, creemos en la potencialidad de las cooperativas para abordar el envejecimiento activo en la empresa, y es que ese mismo potencial se ubica precisamente en sus valores fundamentales, tales como una mayor cercanía hacia sus personas trabajadoras (cooperativistas) y beneficiarias, un mejor conocimiento de los problemas sociales más actuales y una mayor capacidad para que se cubran esas necesidades. Labor que, además actualmente, se completa con facilidades para obtener recursos financieros a fin de poner en marcha las iniciativas empresariales bajo estas formas jurídicas (además de la capitalización del desempleo o subvenciones directas, por asistencia técnica o bonificaciones de los tipos de interés de los préstamos, las sociedades cooperativas pueden también obtener subvenciones si incorporan como socios trabajadores a desempleados mayores de 45 años y si realizan actividades de formación, fomento y difusión de la economía social vinculadas directamente al fomento del empleo ${ }^{25}$.

23 Dicha cooperativa se crea como alternativa a las residencias de la tercera edad y se rige sobre los principios de envejecimiento activo anteriormente citados. De esta manera, se constituye como un complejo residencial y asistencial para personas mayores donde la ayuda mutua, la solidaridad y la empatía son los valores cooperativos fundacionales. Así pues, su premisa primordial es que el $80 \%$ de sus socios deben ser personas autónomas con objeto precisamente de contribuir al envejecimiento activo mediante gente independiente $\mathrm{y}$ autónoma que pueda tener una vejez vitalista y que al mismo tiempo colaboren y ofrezcan un estímulo y un ambiente agradable e integrador al restante $20 \%$ de cooperativistas. Para más información consultar: https://brisadelcantabrico.com/.

24 En esta línea, existen programas como "Vida en plenitud" elaborados y llevados a cabo por las cooperativas en América latina como Coomeva.

25 Los riesgos inherentes al microemprendimiento podrían minimizarse mediante la formación de redes de microempresas que permitan compartir tanto los riesgos, consiguiendo minimizarlos, como maximizar beneficios. (Martín López, 2006). Ley 11/2013, de 26 de julio, de medidas de apoyo al emprendedor y de estímulo del crecimiento y de la creación de empleo (Boletín Oficial del Estado, núm. 179, de 27 de julio de 2013) y Ley $31 / 2015$, de 9 de septiembre, por la que se modifica y actualiza la normativa en materia de autoempleo y se adoptan medidas de fomento y promoción del trabajo autónomo y de la Economía Social. (Boletín Oficial del Estado, núm. 217, de 10 de septiembre de 2015). 


\section{Innovación en materia de gestión de la edad en la empresa}

\subsection{La necesidad de continuar en el mercado de trabajo}

Desde el comienzo del siglo $\mathrm{XXI}^{26}$ la Comisión recomienda vincular el aumento de la esperanza de vida a la edad de jubilación (Comisión Europea, 2009) para afrontar el gran reto del envejecimiento de la población y el déficit de los sistemas de pensiones ${ }^{27}$. De esta forma se viene entendiendo que han de promocionarse las vidas laborales y de cotización más largas y restringirse el acceso a los planes de jubilación anticipada y a otras vías de salida temprana del mercado laboral. También la Estrategia Europa 2020 evidencia la preocupación por las pensiones al suponer una de las mayores partidas de gasto público.

La mayoría de los Estados miembros han adaptado sus sistemas de pensiones para hacer frente al proceso de envejecimiento de la población e intentar garantizar la sostenibilidad del sistema reformando su legislación a fin de considerar toda la vida laboral a la hora de realizar el cálculo de la pensión, con periodos transitorios en la implantación de las modificaciones: Alemania (donde pasan a requerirse los ingresos y cotizaciones de toda la carrera), Finlandia (teniendo en cuenta los salarios medios) o Suecia (el cálculo se realizará sobre todas las cotizaciones efectuadas) (Informe sobre el factor de sostenibilidad, 2013: 7). En la misma línea, las reformas introducidas han implantado medidas para rebajar los índices de actualización tanto de las pensiones como de las cotizaciones necesarias para el cálculo de la pensión. La introducción de nuevas variables en la forma de cálculo se traduce directamente en una rebaja de la cuantía final de las pensiones, y por ello ha sido la técnica mayoritariamente empleada ${ }^{28}$. Por otra parte, las reformas realizadas han establecido un número mayor de años cotizados para tener derecho al $100 \%$ de la pensión. En este sentido, la media europea de años cotizados para acceder a la pensión completa se sitúa en torno a los 40 años cotizados (Informe sobre el factor de sostenibilidad, 2013: 8).

La edad ordinaria de jubilación también ha aumentado en prácticamente todos los países de la UE ${ }^{29}$. En España, la Ley 27/2011, de 1 de agosto, sobre

26 Comunicación de la Comisión al Consejo, al Parlamento Europeo y al Comité Económico y Social, Evolución futura de la protección social desde una perspectiva a largo plazo: Pensiones seguras y viables (COM (2000) 622 final-no publicada en el Diario O ficial).

27 Es al igual que apunta el proyecto SI DRIVE, el reto particular al que la UE se enfrenta actualmente. En otras palabras, las organizaciones se enfrentan al problema de que gran parte de la fuerza de trabajo se aproxima a la edad de jubilación, mientras que al mismo tiempo la disponibilidad de mano de obra cualificada para reemplazar al primer grupo es escasa. Fuente de información: https://www.si-drive.eu/?page_id=19 (Última consulta: 24/08/2017).

28 Mientras que algunos países han congelado la cuantía de las pensiones (como es el caso de Irlanda), otros han variado su método de indexación, sustituyéndolo o bien añadiendo nuevas variables a la forma de cálculo. Éste es el caso de Dinamarca, donde la pensión nacional se revaloriza en función de cómo evolucionen los salarios, o de Luxemburgo, estado en el que las pensiones se revalorizan en función de su IPC y los salarios, si bien prevé que en el caso de que se produzca una situación de insuficiencia financiera, la indexación se hará tan solo en función de la evolución del coste de la vida.

29 Italia ha aumentado la edad de jubilación ordinaria a 66 años para los hombres y a 62 años para las mujeres, pero establece un sistema de aumento progresivo que para el año 2021 situará la edad de jubilación ordinaria para ambos géneros en 67 años. En Francia también se producirá un aumento gradual de las edades ordinarias de jubilación para hombres y mujeres entre 2017 y 2022 (de 65 a 67 años y de 60 a 62 años respectivamente) y en Alemania se producirá otro aumento gradual de 65 a 67 años entre 2012 y 2029 . Holanda aumentará la 
actualización, adecuación y modernización del sistema de Seguridad Social ${ }^{30}$ prevé $^{2}$ el progresivo incremento de la edad de jubilación hasta situarla en el año 2020 en 67 años $^{31}$; la reforma del año 2013 adolece del consenso que sí tuvo la reforma del año 2011. La Ley 23/2013, de 23 de diciembre, reguladora del Factor de Sostenibilidad y del Índice de Revalorización del Sistema de Pensiones de Seguridad Social ${ }^{32}$ establece, con entrada en vigor en enero del año 2019, un nuevo régimen para recalcular la cuantía de las pensiones; el Decreto-Ley 5/2013, de 15 de marzo $^{33}$ pretende favorecer la continuidad en el mercado de trabajo de las personas mayores y promover el envejecimiento activo así como luchar contra la discriminación laboral por razón de la edad. Para ello aborda áreas como la jubilación anticipada y parcial, la compatibilidad entre trabajo y pensión ${ }^{34}$ y la lucha contra la discriminación por razón de edad en el empleo. Respecto a las cláusulas de jubilación forzosa el RDL 3/2012 35 y la Ley 3/2012 (Disposición Final $\left.4^{\circ} .2\right)$ prohíben de forma definitiva su previsión ${ }^{36}$ en la empresa, decisión validada posteriormente por el $\mathrm{TC}^{37}$.

edad a 68 años en 2028, mientras que en Reino Unido las mujeres igualan en 65 años a los hombres para el año 2018 y ambos aumentan a 66 años entre 2018 y 2020. Finalmente, se posibilita la percepción de la pensión de jubilación con los rendimientos del trabajo. Los sistemas del algunos Estados miembros aceptan esta posibilidad sin limitación alguna, como es el caso de Austria, Suecia, Finlandia o Luxemburgo, mientras que otros países de la UE establecen ciertas limitaciones de carácter cuantitativo, como Alemania u Holanda (Informe sobre el factor de sostenibilidad, 2013: 8).

30 BOE núm. 184, de 2 de agosto de 2011. De forma paradójica, la Ley endurece los requisitos para el acceso a la jubilación anticipada al tiempo que incrementa el número de supuestos de anticipación de jubilación. La explicación se encuentra en el Informe de Evaluación y Reforma del Pacto de Toledo de 2011 y en el que se constata que la jubilación anticipada se ha convertido en una fórmula de regulación de empleo (Elorza Guerrero, 2016).

31 La reforma de 2011 fue el resultado del diálogo social y el acuerdo de los interlocutores sociales con el Gobierno. Con ella se perseguía garantizar una protección similar a la actual en momentos de mayor tensión demográfica mediante la contención de la proyección de gasto hasta un umbral -situado en el entorno del 14$15 \%$ del PIB para el año 2050. De este modo, se aseguraría, entre otras cuestiones, una tasa de reposición de las pensiones públicas en el entorno del $70-80 \%$.

32 BOE núm. 309, de 26 de diciembre.

33 Real Decreto-Ley 5/2013, de 15 de marzo, de medidas para favorecer la continuidad de la vida laboral de los trabajadores de mayor edad y promover el envejecimiento activo (Boletín Oficial del Estado, núm. 65, de 16 de marzo).

34 En este sentido, el Gobierno de España ha recordado que tiene la intención de poner en marcha la posibilidad de compatibilizar al 100\% la pensión con el salario (Viernes 21 de diciembre de 2016, www.elcorreo.com). Una medida no necesariamente más beneficiosa para la empresa dado que no siendo obligatoria la jubilación de los trabajadores, si el empleado quiere seguir trabajando y compatibilizarlo con la pensión, la empresa que quiera prescindir de él tendrá que despedirlo o llegar a un acuerdo.

35 De 10 de febrero, de medidas urgentes para la reforma del mercado laboral (BOE núm. 36).

36 BOE núm. 162. Dicha Ley dio la siguiente redacción a la Disposición adicional $10^{\mathrm{a}}$ del ET: Cláusulas de los convenios colectivos referidas al cumplimiento de la edad ordinaria de jubilación: "se entenderán nulas y sin efecto las cláusulas de los convenios colectivos que posibiliten la extinción del contrato de trabajo por el cumplimiento por parte del trabajador de la edad ordinaria de jubilación fijada en la normativa de Seguridad Social, cualquiera que sea la extensión y alcance de dichas cláusulas".

37 La STC de 22 de enero de 2015 (Recurso de Inconstitucionalidad núm. 5610/2012), que resuelve el recurso de inconstitucionalidad interpuesto por el Grupo Parlamentario Socialista y el Grupo Parlamentario de Izquierda Unida en relación a diversos preceptos de la Ley 3/2012, de 6 de julio, entre ellos, el establecimiento de cláusulas de jubilación forzosa, razona "aunque con anterioridad se había señalado la legitimidad de una política de empleo basada en la jubilación forzosa como causa de extinción de la relación laboral, aun cuando suponía una limitación del ejercicio del derecho al trabajo de unos (vertiente individual), se justificaba constitucionalmente en el objetivo de promover el reparto o redistribución del trabajo facilitando así el ejercicio del derecho al trabajo (vertiente colectiva), no cabe sino afirmar ahora que otra politica de empleo basada en la facilitación de la continuidad en el empleo de quienes habiendo superado la edad legal 
Efectivamente, la continuidad en el mercado de trabajo es la premisa básica para afrontar el proceso de envejecimiento de la población pero el envejecimiento activo en el ámbito laboral, partiendo de esa base y para que no quede en saco roto, necesita la puesta en marcha de políticas laborales que fomenten la conservación del empleo en personas de edad avanzada y la reintegración de los trabajadores mayores. Ello, en la aplicación práctica, topa con los estereotipos existentes sobre los trabajadores mayores y la discriminación que aún sufren éstos en el mercado de trabajo.

Según el Eurobarómetro ${ }^{38}$, un $43 \%$ de los ciudadanos españoles opinan que la discriminación por edad está bastante extendida. Un $67 \%$ piensan que cuando una empresa quiere contratar a alguien y puede elegir entre candidatos con las mismas habilidades y cualificaciones, el ser mayor de 55 años es un criterio que puede poner al trabajador en desventaja. Por todo ello al $93 \%$ de los españoles les gustaría que la UE interviniera más que actualmente en la lucha contra el desempleo de hombres y mujeres de hasta 64 años. La situación resulta aún más preocupante si se tiene en cuenta que según el $\mathrm{INE}^{39}$ la reducción del desempleo en el segundo semestre del año 2017 se concentra entre las personas de 25 a 54 años $(314.800$ parados menos) mientras que el paro se mantiene en el grupo de 55 años y más (en la actualidad es del $14,85 \%)^{40}$. También se manifiesta esta preocupación en la "Estrategia Global para el Empleo de los Trabajadores de Más Edad 2012-2014 (Estrategia 55 y más)" ${ }^{41}$. La tasa de desempleo de los trabajadores de edad ha crecido hasta alcanzar cotas muy elevadas y su duración es cada vez más larga (Consejo Económico y Social, 2009). Así, la contratación de los trabajadores a partir de los 45 años es realmente difícil en España porque el factor edad afecta negativamente a los procesos de selección en un 65\% de los casos (Innobasque, 2013: 25). Detrás de esta situación no hay una sola repuesta: el trabajador mayor es más caro (le puede costar aceptar niveles salariales que los jóvenes no rechazan), asume tener derechos laborales y pide que las empresas los respeten, y reivindica

de jubilación desean continuar con su vida laboral activa, con sustracción en consecuencia de esta materia a la potestad negociadora de los representantes de los trabajadores y empresarios, encuentra perfecto acomodo también a los mandatos y objetivos constitucionales". Apostilla el TC que en el contexto socioeconómico y del mercado de trabajo en el que se inserta dicha medida, caracterizada por una situación de crisis económica, el objetivo de estimular la continuidad del trabajador en su puesto de trabajo sirve también para garantizar la protección de un interés general prevalente como es la sostenibilidad del sistema de pensiones y la viabilidad del Sistema Nacional de la Seguridad Social. Sin embargo ¿Qué es más razonable, pues para el ciudadano, esperar a la puesta en escena eficaz de esas prometidas maravillas del envejecimiento activo o aprovechar la primera oportunidad que le brinde el ordenamiento para acceder a la pensión de retiro en un contexto de crisis intensa con constantes dudas sobre el futuro del empleo, sus condiciones y también las pensiones? Las autoridades públicas, mantienen un discurso oficial pero practican lo opuesto. Piénsese en el compromiso de ayudas públicas para todos los trabajadores de estiba portuaria que voluntariamente rescindan su contrato por razón de la edad. (Molina Navarrete, 2017: 11).

38 Datos anuales del Eurobarómetro 2016 y del Eurobarómetro del segundo semestre de 2017. En: http://www.europarl.europa.eu/pdf/eurobarometre/2017/2019ee/two_years_until_the_2019_european_election s_es_es.pdf. (Última visita 19/09/2017).

39 Datos relativos al año 2017 por edad. www.ine.es (Última visita 18/09/2017).

40 Tal y como se recoge en el SI DRIVE eldesempleo sigue siendo el principal desafío frente al que las políticas de innovación social en el empleo se encuentran a nivel europeo y mundial. Fuente de información: https://www.si-drive.eu/wp-content/uploads/2015/12/SI-DRIVE-Policy-Brief-Employment-2015.pdf (Última consulta: 24/08/2017).

41 Aprobado por el Consejo de Ministros de 28 de octubre de 2011 y publicado por Resolución de 14 de noviembre de 2011 de la Secretaría de Estado de Empleo (BOE de 24 de noviembre). 
su derecho a un equilibrio entre vida y trabajo, un hándicap frente a trabajadores jóvenes más baratos, más dóciles y que aceptan contratos efímeros. Esa huida de la precariedad laboral no puede, desde luego, abocarles al desempleo.

Lo cierto es que no es fácil la supervivencia de los trabajadores de edad avanzada en las empresas porque éstas no consiguen ver el talento oculto y los valores que los trabajadores aportan: responsabilidad, seriedad, experiencia y control emocional, entre otros. Invertir y aprovechar el potencial de las personas mayores necesita de la implementación de sistemas de gestión de la edad y sobre todo, necesita de empresas que crean y confien en ellos.

\subsection{Medidas de gestión de la edad en la empresa}

Si realmente se quiere incorporar y mantener a trabajadores mayores en las empresas hay que implementar medidas adecuadas de gestión de la edad, lo que necesariamente conlleva innovación en las relaciones laborales e imaginación en la gestión de los recursos humanos facilitando la transferencia del conocimiento acumulado, evitando la discriminación y permitiendo la renovación de la plantilla de la empresa ${ }^{42}$. Las medidas de gestión de la edad conllevan implícito un deber de prevención para que la vulneración de los derechos de las personas mayores no llegue a producirse, línea que siguen los Principios Rectores de Naciones Unidas sobre las Empresas y los Derechos Humanos al establecer "la diligencia debida" que, básicamente, supone la responsabilidad de las empresas de adoptar acciones para prevenir la violación de los derechos fundamentales dentro de su ámbito de organización y dirección (Velázquez Fernández, 2015) ${ }^{43}$.

Las medidas de gestión de la edad se inscriben en el marco de la llamada "gestión de la diversidad" (Innobasque, 2013: 12) en la empresa. Al margen de la edad de jubilación legal establecida no es fácil determinar cuál es la edad concreta en la que la persona trabajadora se considera de edad avanzada y pasa a ser sujeto en la aplicación de las mismas. En este sentido, las edades de referencia varían según los contextos culturales, de desarrollo económico del país donde se encuentran los trabajadores y de actividad concreta donde prestan sus servicios, pues no es posible señalar una edad única y general, al margen de éstas y otras circunstancias que concurren en la determinación de las condiciones del trabajador (Urrutikoetxea, 2011: 20). Podemos afirmar que existen 3 variables de edad para el desarrollo de las medidas de gestión de la edad, los 45 , los 55 y los 65 años $^{44}$.

42 En el Plan "cuidamos la experiencia" de Gas Natural Fenosa, por ejemplo, se compromete la contratación de jóvenes en los mismos puestos ocupados por trabajadores mayores en un plan en el que los jóvenes se incorporan a una formación en el que los trabajadores mayores realizan funciones tutoriales con ellos transmitiéndoles su experiencia al mismo tiempo que van preparando su retirada del mercado de trabajo.

43 Sin carácter vinculante, estos principios, como manifestación del llamado soft-law, son un referente internacional en la aplicación de los derechos fundamentales que cuenta con el consenso de la comunidad internacional. Pero además, existe otro ámbito más cercano así como el de las propias organizaciones que, tal y como concluye el SI DRIVE, desde su seno trata de encontrar sus propias soluciones innovadoras para lidiar con los problemas de la organización del trabajo. Podemos hablar así de la importancia de la autorregulación dentro de las organizaciones para establecer medidas innovadoras socialmente. Fuente de información: https://www.si-drive.eu/?page_id=19 (Última consulta: 24/08/2017).

44 El primer referente de los 45 años se da porque es cuando las trabajadoras y trabajadores son "aún una generación con capacidad de poder e influencia en el sentido de que muchos de sus miembros ocupan posiciones clave en las capas elevadas de la sociedad" según autores como Cerrato, Ugarteburu e Ibarretxe 
Concretamente, algunos estudios ${ }^{45}$ concluyen que es en esas edades cuando los beneficios son evidentes tanto para las empresas como para los trabajadores: aumenta la competitividad; promueve el "feedback intergeneracional" y la transmisión del conocimiento; maximiza el potencial de los senior; retiene el talento; desarrolla una mayor motivación y bienestar en el trabajo; aumenta la formación, desarrollo y promoción individual; facilita la adquisición de responsabilidades por el reconocimiento de su valía personal; optimiza al máximo sus experiencias para trasladarlas a otras personas de la empresa y ayuda a afrontar mejor un envejecimiento saludable ${ }^{46}$. Así pues, en contraposición con los planes de jubilación anticipada que se utilizaron a finales de los setenta y durante los ochenta, la apuesta ahora son políticas de empleo en las que el objetivo mínimo sea que la edad de salida del mercado laboral de los trabajadores coincida con la edad de jubilación (Martín López, 2006).

En algunos países de la UE la gestión de la edad en la empresa está ya prevista en las normas o convenios colectivos. Así por ejemplo, en Francia, las normas de Seguridad Social aprobadas en el año 2009 incentivan a las empresas de más de 50 trabajadores para que lleven a cabo al menos tres medidas de gestión de la edad dentro de las seis previstas en las normas de Seguridad Social a favor de los mayores de 55 años $^{47}$, y en Bélgica el Convenio núm. 104 de 27 de junio de 2012 establece la obligación de las empresas de 45 o más trabajadores de realizar un plan de medidas de gestión de la edad (Velázquez Fernández, 2015).

En el caso de España, las políticas de empleo y el envejecimiento en el ámbito laboral podrían resultar en ocasiones contradictorias ya que se mueven entre la necesidad u obligación de mantener a unos mayores (a los que no se quiere) en el mercado de trabajo ${ }^{48}$ y el impulso de políticas de empleo para la contratación de

(2011). Además, el informe europeo Working conditions of an ageing workforce establece que la edad clave para intervenir y limitar la salida anticipada del mercado laboral de las personas trabajadoras maduras comienza a partir de los 45 años. El segundo referente, el de los 55 años se establece porque es a esta edad cuando las personas trabajadoras se comienzan a considerar personas trabajadoras de edad avanzada ya que se ubican en el tramo siguiente antes de la tercera edad en opinión de autoras como Sánchez-Urán Azaña (2011). Y finalmente, el tercer referente de los 65 se ha percibido como "barrera de la ancianidad", es decir, se considera el momento en el que la persona trabajadora abandona el mercado laboral, aunque este umbral puede verse ampliado en los casos en los que las personas trabajadoras decidan permanecer en el mercado de trabajo y jubilarse con una edad mayor.

45 Así como el realizado por la MUTUA NAVARRA, Gestión de la edad en la empresa. Reto y oportunidad, Pamplona, Colección Recomendaciones para una empresa saludable, 2013, pág. 77-79. Son numerosas las guías y recomendaciones para una gestión adecuada de la edad en las empresas en consonancia con lo establecido a nivel europeo por el COMITÉ DE PROTECCIÓN SOCIAL Y COMITÉ DE EMPLEO, Declaración del Consejo sobre el Año Europeo del Envejecimiento Activo y de la Solidaridad Intergeneracional: estrategia futura, 16592/12 SOC 948 SAN 289.

46 En este sentido, hay ejemplos de empresas en las que han conseguido reducir sus costes de contratación al adoptar una política positiva de contratación en relación con la edad. Como buena práctica, Natural Gas Service (empresa de instalación y servicio de gas) ha conseguido notables ventajas de unas prácticas de contratación no discriminatorias por razón de edad. Así, un nuevo empleado de más de 50 años ha liderado el desarrollo de un nuevo sistema de aseguramiento de la calidad utilizando la experiencia obtenida en empleos previos. (Innobasque, 2013: 12).

47 El Decreto 2009/560 sobre el contenido y valoración de los acuerdos y planes de acción a favor de los trabajadores de más edad reforma en este sentido los artículos 138-25 y siguientes del Código de Seguridad Social de Francia.

48 Con medidas que implícitamente, como se ha explicado en este trabajo, avalan la salida del mercado laboral. 
jóvenes y renovación de la plantilla de la empresa ${ }^{49}$. En la actualidad, no sólo no hay relevo generacional sino que ambos colectivos de edad, jóvenes y mayores, padecen dificultades para insertarse y mantenerse, respectivamente, en el mercado de trabajo y cargan sobre ellos los estereotipos, no resueltos socialmente, de su inexperiencia y bajo rendimiento respectivamente (Sánchez-Urán Azaña' ${ }^{2011}$ ). El derecho de los mayores a permanecer en el puesto de trabajo, el aprovechamiento de sus conocimientos y experiencia y las políticas de contratación de trabajadores más jóvenes han de analizarse de modo conjunto ${ }^{50}$. Es clarificadora la posición del Gobierno de Gran Bretaña que ha impulsado medidas desde la denominada población activa multigeneracional, sensibilizando a los empresarios para que el empleo de los trabajadores de edad avanzada no sea visto como una batalla "jóvenes versus mayores" por el empleo sino, por el contrario, como una oportunidad para interrelacionar a ambos a través de la formación, el mentoring o el trabajo compartido orientados hacia un retiro flexible y gradual de los trabajadores de más edad, a través de planes empresariales de jubilación que permitan también el reemplazo flexible y gradual ${ }^{51}$.

En este escenario, en España, como hemos dicho, al contrario que ocurre con ámbitos como la igualdad o discapacidad, no hay una obligación legal de implementar medidas de gestión de la edad en las empresas, pero sí existen planes y diferentes manuales de buenas prácticas empresariales que identifican experiencias empresariales innovadoras en materia de gestión de la edad ${ }^{52}$. Para una adecuada implementación de las medidas de gestión de la edad en la empresa es necesario en primer lugar, realizar un diagnóstico de situación para analizar tanto la posición como las condiciones de la organización y de sus personas trabajadoras. Y en segundo lugar, determinar las medidas necesarias para una

49 ¿Para qué hacer de la gestión de la seguridad y salud en el trabajo desde el enfoque del envejecimiento activo uno de los ejes de esa política en el futuro sibuena parte de los empresarios y trabajadores coinciden en hacer de la jubilación un objetivo común -III Acuerdo para el Empleo y la Negociación Colectiva- aunque haya que aportar más recursos al Erario Público -disposición adicional $16^{\circ}$ Ley 27/2011-? Cierto, más preguntas que respuestas que revelan no solo las contradicciones y las paradojas de esta nueva dimensión del problemaconflicto intergeneracional vinculado a la edad, en este caso de los trabajadores, sino las propias incertidumbres, lagunas y antinomias del Derecho español en esta materia, que viene navegando entre dos aguas, sin rumbo cierto (Molina Navarrete, 2015:124).

50 Para ello, los contratos de relevo, cuyos requisitos se han visto endurecidos tras la llamada reforma del mercado de trabajo en el año 2012, u otro tipo de políticas similares que incluyan un enfoque intergeneracional son una buena forma de facilitar el acceso de los jóvenes al mercado de trabajo y la salida escalonada de trabajadores de más edad, minimizando los aspectos negativos de quién sale (reducción parcial de salario, adaptación a circunstancias de salud o personales cambiantes...) y favoreciendo la adquisición de experiencia profesional de los más jóvenes (Innobasque, 2013: 43).

51 Fuente de información: Department for Work and Pension, UK: Employing Older Workers. An employer's guide to today's multi-generational workforce, 2013, www.gov.uk.

52 España cuenta con el Real Decreto-ley 5/2013, de 15 de marzo, de medidas para favorecer la continuidad de la vida laboral de los trabajadores de mayor edad y promover el envejecimiento activo (Boletín oficial del Estado, núm. 65, de 16 de marzo de 2013), el Libro Blanco sobre Envejecimiento Activo publicado por el Ministerio de Sanidad, Política Social e Igualdad en 2011, o la Estrategia Vasca de Envejecimiento Activo 2015-2020 (http://envejecimiento.csic.es/documentos/documentos/ESTRATEGIAVASCA 2015.pdf). Y entre los manuales de buenas prácticas podemos encontrar: INNOBASQUE Agencia vasca de la innovación. Guía para la gestión de la edad en las organizaciones de Euskadi: Doce propuestas de aplicación en las organizaciones y tres recomendaciones para las administraciones, 2013; IESE BUSINESS SCHOOLUNIVERSIDAD DE NAVARRA. Cómo gestionar personas en una sociedad madura, 2015; O FEMEVAL Federación Empresarial Metalúrgica Valenciana. La buena práctica en gestión de la edad: aplicación de la gestión de la edad en la empresa, 2006. 
adecuada política de gestión de la edad desde la contratación inicial en la organización hasta la salida, pasando por la optimización de las carreras profesionales. El diagnóstico requiere una reflexión acerca de la edad del personal, es decir, hay que situar la edad de cada persona trabajadora de la organización ${ }^{53}$. Como resultado del diagnóstico que concretará la composición generacional de la organización se podrá proceder a la determinación y clasificación de medidas de gestión de la edad ${ }^{54}$, que en este estudio dividiremos en tres bloques generales: las medidas de acceso, las medidas de mantenimiento y las medidas de salida (Innobasque, 2013: 21).

Entre las medidas de acceso encontramos las relativas a la no discriminación en la contratación y a la diversificación de la edad de la plantilla. En aras de no discriminar a las personas mayores con una experiencia dilatada frente a la incorporación de personas jóvenes (cuya capacidad de adaptación y formación dentro de la organización está más valorada) los procesos de selección pueden basarse en la valoración del puesto de trabajo realizada y en las competencias, habilidades y aptitudes que este requiere; además, durante los mismos conviene hacer uso de instrumentos que no fijen el factor edad como determinante para la obtención del puesto de trabajo, y para ello, debieran analizarse los CV desde una óptica donde no se evalúen la edad ni el sexo ${ }^{55}$.

En lo que respecta a las segundas, resulta beneficioso que toda organización cuente con una plantilla equilibrada en edad ya que ello fomenta un valor añadido así como un mejor conocimiento de la clientela, o incluso de los productos y servicios del mercado. Entre las medidas que las organizaciones podrían tomar en este sentido están ${ }^{56}$ : el anuncio de vacantes haciendo hincapié en las características del puesto de trabajo (como anteriormente hemos mencionado) pero también haciendo uso de asociaciones o colegios profesionales que trabajen de primera

53 El diagnóstico inicial al que hacemos alusión también es considerado como una de las buenas prácticas identificadas para gestionar la edad de las personas trabajadoras (Femeval, 2006).

54 Todas las medidas referidas a continuación se ubican sobre los 8 ejes principales que el estudio de EUROFOUND-A guide to good practice in age management recoge como dimensiones fundamentales para la gestión de la edad. (Eurofound, 2016: 7).

55 Es concretamente sobre las medidas y condiciones de acceso al empleo donde se circunscribe una especial protección jurídica a las personas trabajadoras de mayor edad por medio de la Directiva 2000/78 del Consejo, relativa al establecimiento de un marco general para la igualdad de trato en el empleo y la ocupación (Diario Oficial de las Comunidades Europeas, núm. 303, de 2 de diciembre de 2000).

Por lo que podemos afirmar que las medidas recogidas en este apartado son esenciales para garantizar un marco de actuación para luchar contra la discriminación que las personas mayores sufren en el empleo por motivo de edad (Ríos Mestre, 2017). Además la prevención de cualquier tipo de vulneración de los derechos laborales de las personas de mayor edad en el trabajo encuentra una configuración paralela a los planes de igualdad que por razón de género son de carácter obligatorio en las empresas de más de 250 personas trabajadoras, lo cual está regulado en la Ley Orgánica 3/2007, de 22 de marzo, para la igualdad efectiva de mujeres y hombres (Boletín Oficial del Estado, núm. 71, de 23 de marzo de 2007) (Velázquez Fernández, 2015: 76).

56 La diversificación de la edad de la plantilla es sumamente relevante ya que a pesar de que a nivel organizacional existen corrientes que defienden que las personas trabajadoras de mayor edad conllevan más gastos para las organizaciones la experiencia real e individual de las mismas nos muestra que con la contratación de personas trabajadoras de mayor edad se gana en experiencia y habilidades; también ahorran en futuros gastos cuando se invierte en la investigación de la promoción de habilidades y la salud en el empleo para la plantilla de edad avanzada; Además al retener a esta parte de la plantilla en la organización se consigue evitar la costosa pérdida de habilidades y se puede aumentar la cuota de mercado; finalmente, si se invierte en la diversificación de edad de la plantilla se incrementan tanto la solidaridad intergeneracional como la motivación laboral de todas las personas trabajadoras (Eurofound, 2016:5). 
mano con personas de edad con experiencia laboral o la inclusión de personas trabajadoras de todas las edades en cada grupo profesional, garantizando la transferencia del conocimiento y enriquecimiento del grupo por medio de grupos heterogéneos ${ }^{57}$.

Las medidas de mantenimiento se dividen en varios ámbitos el de promoción y desarrollo profesional, el de transferencia del conocimiento, el de seguridad y salud en el ámbito laboral, y el de reducción y flexibilización del tiempo de trabajo ${ }^{58}$. La finalidad del primer grupo de medidas es promover y facilitar el desarrollo profesional de las personas mediante el acceso a la formación a través de, entre otras, el fomento de la participación de las personas trabajadoras de más de 45 años en las sesiones formativas, adaptando éstas a sus necesidades e intereses específicos mediante el diseño de un plan de formación dirigido a personas que ya son competentes en su actividad laboral. La revisión de las carreras profesionales resulta una medida muy relevante ya que la organización debe conocer a medio camino cuales son las necesidades, las expectativas o las carencias laborales que detectan las personas trabajadoras respecto a su carrera, y a su vez, hacerles saber las oportunidades que surjan en este ámbito y cómo conseguirlas. Y es que las organizaciones deben partir del principio general de que todas las personas trabajadoras tienen que contar con las oportunidades necesarias para mejorar en su situación profesional como fruto de su experiencia y los conocimientos adquiridos (Velázquez Fernández, 2015: 81). En este punto, es importante destacar que la promoción profesional a nivel horizontal de las personas de mayor edad puede coadyuvar a una mayor satisfacción personal, al desarrollo de nuevas capacidades, nuevas responsabilidades de trabajo y al mantenimiento de la motivación en el ámbito profesional de manera que se aleje de la monotonía ${ }^{59}$; por otro lado, las medidas de transferencia del conocimiento se establecen con el fin de que las organizaciones no pierdan conocimientos cuando se da un caso de jubilación entre su plantilla. Para lograrlo resulta necesario elaborar un mapa de conocimiento que prevea la futura pérdida del capital humano de la persona que deja la organización y la intente evitar. Así, el mapa debe recoger cuáles son las fuentes y los activos de conocimiento de la organización y promover la cultura y el entorno del conocimiento; otra de las medidas de transferencia es el mentoring. Éste consiste en el asesoramiento, coaching o formación de una persona experimentada en la organización al personal más joven o a personas en determinados puestos de trabajo y puede hacer que la experiencia de las personas trabajadoras de mayor edad sea útil y obtenga el mayor rendimiento posible entre las personas que componen la organización. Para esta transferencia del conocimiento es

57 La guía de gestión de la edad elaborada por FEMEVAL hace hincapié en la diversificación de la edad de la plantilla concretamente mediante la incorporación de trabajadores y trabajadoras de más de 45 años, estrechamente relacionada con la incorporación de una fuerza productiva de trabajo especialista y experimentada (Femeval, 2006: 17).

58 También autoras como Martín López inciden en la importancia de la adaptación de contenidos del puesto de trabajo, el aprovechamiento del conocimiento o la formación continua de las personas trabajadoras como vía para gestionar la edad en el empleo para lo cual resulta un factor indispensable la implicación de toda la plantilla, de otro modo, su éxito no está garantizado (Martín López, 2006).

59 También esta medida es desarrollada en la guía de buenas prácticas del INSTITUTO NACIONAL DE SEGURIDAD E HIGIENE EN EL TRABAJO NTP 367: Envejecimiento y trabajo: la gestión de la edad. Ésta hace hincapié en la importancia del establecimiento y los ajustes de los planes de carrera, centrándolos en este caso en la segunda fase de la vida laboral de las personas trabajadoras que tiene lugar a partir de los 40 años. 
indispensable (como hemos mencionado anteriormente) que la organización constituya equipos de trabajo con personas de edades mixtas ${ }^{60}$.

Las medidas de seguridad y salud en el ámbito laboral son necesarias para evitar la pérdida de capacidad de las personas trabajadoras de edad. Así pues, es importante que las organizaciones cuenten con medidas de prevención en lo referente a la salud física y psicológica de la plantilla, además de medidas destinadas a reducir la penosidad o el riesgo que conllevan ciertas actividades profesionales, en su caso (Velázquez Fernández, 2015: 83). Las medidas enfocadas al cuidado de la salud son, a modo de ejemplo las siguientes: la incorporación y el uso de las nuevas tecnologías en la organización para desarrollar actividades pesadas o físicas ${ }^{61}$; el establecimiento de medidas de adaptación de funciones y condiciones de trabajo, que a diferencia de la medida expuesta en el punto anterior se refieren, entre otras, a la posibilidad de que la plantilla de más edad lleve a cabo turnos de menor duración o con un margen de descanso más amplio, tengan preferencia a la hora de elegir horario, sean sustituidos en las actividades que engrosan un mayor esfuerzo físico por personal más joven pasando a realizar estos trabajos de supervisión donde su experiencia es un potencial, e incluso que la organización prevea un cambio de funciones para estos después de realizar un periodo de nueva profesionalización; además de realizar sesiones formativas y de sensibilización a la plantilla en temas como el estrés, la conciliación del sueño e incluso la organización de cursos para combatirlos ${ }^{62}$.

En lo que respecta a las medidas de reducción y flexibilización del tiempo de trabajo recordar que son fundamentales para que las personas trabajadoras de mayor edad puedan afrontar condicionantes relacionados con la edad. Para ello, las organizaciones pueden establecer medios de trabajo basados en las nuevas tecnologías, como el teletrabajo, a fin de permitir no tener que trasladarse físicamente y a diario a la organización y poder realizar la prestación laboral desde un lugar diferente, normalmente su propio domicilio; o también pueden establecer nuevos horarios continuos e ininterrumpidos de 8-15 h.

El tercer grupo de medidas gira en torno a las medidas de salida y se concretan en el uso del contrato de relevo y la jubilación parcial conjuntamente. Estas medidas permiten que la transición de la actividad a la jubilación se enfoque hacia la optimización de su productividad y la transferencia de su conocimiento. Sin embargo, como se ha dicho con anterioridad, las últimas reformas laborales han

60 En este sentido, la guía de FEMEVAL destaca la importancia de las funciones de mentoring tanto en habilidades para el trabajo como en otros aspectos menos identificables a nivel laboral, que sin embargo, harán que las nuevas personas trabajadoras tengan una formación completa y una guía continua en su integración al trabajo, así como, en la cultura de la organización o el clima organizacional (Femeval, 2006: 13).

61 Así como se ha detectado en el SI DRIVE entre las políticas impulsoras desde la innovación en el empleo, se detecta la digitalización de la actividad laboral, la cual puede suponer que estas tecnologías crucen los límites del tiempo de trabajo productivo y difuminen la barrera entre trabajo y vida privada, por lo tanto, la incorporación de las mismas debe hacerse estableciendo un límite en este sentido. Fuente de información: https://www.si-drive.eu/wp-content/uploads/2015/12/SI-DRIVE-Policy-Brief-Employment-2015.pdf (Última consulta: 24/08/2017).

62 Las medidas mencionadas están íntimamente vinculadas a problemas del envejecimiento derivados de la salud, que si bien exigen en primer lugar y a nivel individual que toda persona trabajadora vele por su salud, también corresponde en segundo lugar a la organización vigilar el estado de salud de la plantilla con trabajos con altas demandas físicas o mentales. (INSTITUTO NACIONAL DE SEGURIDAD E HIGIENE EN EL TRABAJO NTP 367: Envejecimiento y trabajo: la gestión de la edad). 
dificultado su uso en pro de una necesaria continuidad en el mercado de trabajo sin reparar en que precisamente, son medidas de fomento del envejecimiento activo. Otra posibilidad es el establecimiento de bancos de tiempo o reducción del tiempo de trabajo sin pérdida de salario.

\section{La gestión de la edad en el ámbito del cooperativismo en la comunidad autónoma de Euskadi}

A efectos de conocer la implementación de las medidas de gestión de la edad identificadas en este estudio y comprobar si existe preocupación o no por ello se ha realizado una encuesta en el ámbito del cooperativismo vasco $^{63}$. Entre las medidas de gestión de la edad en el acceso al empleo el 50\% de las cooperativas encuestadas dicen tener en cuenta la edad de las personas para acceder a un puesto de trabajo, pero afirman no tener preferencias para la contratación de personas jóvenes, de hecho, el 100\% de ellas manifiesta que en las contrataciones de nuevo personal prevalece la motivación y capacidad humana de las personas frente a su CV. Lo cierto es que actuar de otra forma constituiría una discriminación por razón de edad. Tampoco se ha encontrado ningún caso en el que se apliquen medidas de gestión de la edad en el acceso al empleo, como el uso de instrumentos que omitan y no evalúen la edad de las personas a contratar. El 38\% confirma que resulta muy beneficioso tener una plantilla equilibrada en lo que respecta a la edad y por esta misma razón utilizan asociaciones y colegios profesionales en sus procesos de selección para incorporar nuevas personas con experiencia laboral previa y dilatada, que a su vez, puede servir como medida de transferencia del conocimiento entre sus integrantes. Respecto a esta cuestión sólo el número de personas mayores contratadas avalaría verdaderamente, en su caso, la afirmación. En lo que concierne a las medidas de gestión de la edad en el mantenimiento del empleo, el 62,5\% cuenta con planes de formación individuales y consecuentemente, realizan revisiones sobre las carreras profesionales y la satisfacción profesional del personal. Por consiguiente, ese mismo porcentaje de las cooperativas vascas encuestadas destina parte de sus esfuerzos a la detección de las necesidades y expectativas de su plantilla con el fin de continuar ofreciendo posibilidades formativas. Sin embargo, sólo el $25 \%$ identifica las posibilidades de transferencia del conocimiento por parte de su plantilla más veterana hacia las nuevas incorporaciones mediante técnicas como el mentoring o el establecimiento de mapas de conocimiento que permiten averiguar sus fuentes internas de conocimiento y promoverlas. De los resultados obtenidos concluimos que la formación continua es una preocupación en el ámbito del cooperativismo vasco

63 El método de estudio utilizado ha sido la investigación cualitativa. Para ello hemos recogido información mediante entrevistas con las personas responsables de Recursos Humanos de 8 cooperativas de la CAE. Para la elección de las cooperativas encuestadas se ha buscado una muestra significativa que recoge cooperativas de diferentes sectores económicos (entre ellos, servicios, eléctrico-electrónico, componentes mecánicos, mueble y madera, máquina-herramienta y agroalimentario) y de diferentes tamaños en función de su número de socios/as. Así, un 25\% de las cooperativas encuestadas tiene de 0-10 trabajadores/as; otro 25\% de 10-100 trabajadores/as; otro 25\% de 100-500 trabajadores/as; y finalmente, otro $25 \%$ más de 500 trabajadores/as. Del total de personas trabajadoras de las cooperativas encuestadas, el 38\% de trabajadores/as tiene más de 45 años; el 8,7\% de trabajadores/as tiene más de 55 años; y el 0,05\% tiene más de 65 años. 
encuestado, aunque el conocimiento de los mayores no es considerado aún un valor añadido en la organización. Las cooperativas vascas no son verdaderamente conscientes de su potencial para gestionar la edad de sus trabajadores y socios, es decir, para garantizar su permanencia y plena participación en el trabajo. Teniendo en cuenta que ya cuentan con estas medidas y su implementación no les exige recursos económicos adicionales debieran introducir una nueva óptica en las mismas para favorecer el envejecimiento activo de su personal. Por otra parte, en lo que respecta a las medidas de seguridad y salud en el empleo tendentes a evitar la pérdida de capacidad de las personas mayores, el 75\% reconoce utilizar sistemas tecnológicos para evitar o disminuir el trabajo físico excesivo de su plantilla, sin embargo, no fomentan la reorganización del tiempo de trabajo de las personas de mayor edad (la elección de turno trabajo cuando se trabaja mediante dicho sistema), ni los cambios funcionales para que la plantilla de mayor edad pueda ocupar trabajos de menor intensidad física. De esta manera, es mínimo el porcentaje de cooperativas encuestadas (el 12,5\%) que registra una actividad preventiva específica para las personas de más de 45 años. En cuanto a las medidas de reducción y flexibilización del tiempo de trabajo, sólo el 25\% muestra la utilización del teletrabajo o trabajo a distancia. No obstante, sus principales razones de uso son la conciliación de la vida personal, familiar y profesional (que normalmente se suele dar entre trabajadores/as más jóvenes) o el ahorro de costes de producción, y no el establecimiento de medidas para la transición a la jubilación. $Y$ es que ni siquiera se prevé la jornada laboral continua como medida de gestión de la edad durante la última etapa profesional. En este caso, vemos que cuando se trata de medidas específicas o exclusivas en función de la edad no encontramos ejemplos de su implementación en las cooperativas encuestadas. Por otro lado, sí existen medidas de salida para la gestión de la edad, así como la jubilación parcial, pero estás son mínimas dentro del cooperativismo vasco encuestado ya que sólo está presente en el 38\% de ellas y además, ninguna lo complementa con nuevos contratos de relevo, por lo que no se da la oportunidad de que las personas mayores realicen labores de mentoring y transmitan su conocimiento al nuevo personal ${ }^{64}$. Creemos que la no utilización de esta medida se debe por un lado, a la no contemplación del conocimiento adquirido por las personas mayores como un potencial a explotar, y por el otro, a la desincentivación de su uso por la legislación laboral.

\section{Conclusiones}

Tradicionalmente la innovación ha estado ligada al ámbito tecnológico e industrial, sin embargo, durante los últimos años hemos sido testigos del afianzamiento de su aspecto social. De esta forma, la innovación social es clave para dar respuesta a los

64 Finalmente, ninguna de las cooperativas entrevistadas visualizan los posibles procesos de regulación de empleo que pudieran surgir como vía para reducir el tiempo de trabajo productivo de los mayores pero manteniendo sus condiciones laborales, y es que sería factible pero también incoherente, la idea de llevar a cabo la reducción de la actividad laboral sin dañar las condiciones salariales y derechos sociales futuros de las personas trabajadoras ante una situación económica grave de la cooperativa. 
nuevos retos sociales, entre ellos, el envejecimiento de la población y en concreto, de la población trabajadora.

En efecto, el envejecimiento de la población y la decreciente tasa de natalidad constituyen un fenómeno de gran preocupación en la sociedad europea, con el inminente riesgo de que la futura mano de obra especializada se reduzca a un ritmo desmesurado al mismo tiempo que una cada vez más acuciante necesidad de que se alargue la edad de jubilación hace que la empresa tenga que contar con trabajadores mayores. Las empresas deben ser conscientes de esta situación y el papel de los Estados ante este reto es también indiscutible. Invertir y aprovechar el potencial de las personas mayores necesita de la implementación de sistemas de gestión de la edad y sobre todo, necesita de empresas que creen y confíen en ellos.

El cooperativismo tiene especial responsabilidad en la promoción de estas medidas dado que dos de sus más característicos elementos distintivos son, su propio compromiso para con el desarrollo sostenible de la comunidad, y su compromiso de trabajar por y para las personas. Sin embargo, los resultados del análisis realizado en el ámbito del cooperativismo vasco nos demuestran que aún no es una preocupación real. No hemos encontrado ejemplos de medidas innovadoras para una adecuada gestión de la edad en las cooperativas y si bien algunas de ellas, como los planes de formación individuales, las revisiones de carrera profesional para medir la satisfacción o el uso de nuevas tecnologías podrían sin duda alguna ser utilizadas para una adecuada gestión de la edad, las cooperativas vascas encuestadas no hacen, a día de hoy, uso de ellas con esta finalidad.

\section{Referencias Bibliográficas}

Alvarado García, A. y Salazar Maya, Á. M. (2014) Análisis del concepto de envejecimiento. Gerokomos, No 25, Vol. 2, pp. 57-62.

Buesa Blanco, M. y Molero Zayas, J. (1996) Innovación y diseño industrial: evaluación de la política de promoción del diseño en España. Madrid: Cívitas.

Cerrato Allende, J., Ugarteburu Gastañares, I. e Ibarretxe Zorriketa, R (2011) Factores psicosociales de trabajadoras y trabajadores veteranos y mercado laboral: el envejecimiento activo. Lan Harremanak, $\mathrm{N}^{\mathrm{O}}$ 24, pp. 55-67.

CONSEJO ECONÓMICO Y SOCIAL (2009) Envejecimiento activo: el empleo de los trabajadores de edad avanzada. Revista Cauces, Cuadernos del CES, № 9, pp. 28-39.

COMISIÓN EUROPEA (2009) Sustainability Report. Bruselas: Servicio de Publicaciones de la Dirección General de Asuntos Económicos y Financieros.

COMISIÓN EUROPEA (2011) How to promote active ageing in Europe. EU support to local and regional actors. Bruselas: Oficina de Publicaciones de la Comisión Europea.

COMISIÓN EUROPEA (2012) Ageing Report: economic and budgetary projections for the UE-27 member states, 2008-2060. Bruselas: Oficina de Publicaciones de la Comisión Europea.

CCOO (2016) Observatorio Social de las Personas Mayores para un envejecimiento activo. Madrid: Federación de Pensionistas y Jubilados de CCOO.

Elorza Guerrero, F. (2016) La descentralización de la negociación colectiva en relación a la protección social complementaria sobre jubilación en España. Relaciones Laborales y Derecho del Empleo, № 1, pp.1-27. 
EUROFOUND (2016) A guide to good practice in age management. Ed: Dinamarca.

FEMEVAL (2006) Federación Empresarial Metalúrgica Valenciana. La buena práctica en gestión de la edad aplicación de la gestión de la edad en la empresa, http://www.femeval.es/proyectos/ProyectosAnteriores/Sinnovaciontecnologia/Paginas/ GestiondelaEdad.aspx $(24 / 08 / 2017)$.

Giro Miranda, J. (2006) Envejecimiento Activo, envejecimiento en positivo. Logroño: Universidad de la Rioja (Servicio de publicaciones).

IESE BUSINESS SCHOOL-UNIVERSIDAD DE NAVARRA (2015) Cómo gestionar personas en una sociedad madura, http://www.iese.edu/research/pdfs/ST-0391.pdf $(24 / 08 / 2017)$.

Iimarinen, J. (2012) Promoción del envejecimiento activo en el trabajo. Agencia Europea para la Seguridady Salud en el Trabajo, pp. 1-8. https://osha.europa.eu

INFORME SOBRE EL FACTOR DE SOSTENIBILIDAD (2013) http://www.elconfidencialdigital.com/resources/files/2013/5/17/1368796975794InfoPen siones.pdf $(16 / 03 / 2017)$.

INNOBASQUE (2010) Conclusiones WOKA sobre envejecimiento positivo, https://issuu.com/innobasque/docs/woka_envejeciendo_en_positivo_innobasque (24/08/2017).

INNOBASQUE (2013) Guía para la gestión de la edad en las organizaciones de Euskadi, http://www.carm.es/ctra/cendoc/haddock/16214.pdf (24/08/2017).

INSTITUTO NACIONAL DE SEGURIDAD E HIGIENE EN EL TRABAJO (1995) NTP 367: Envejecimiento y trabajo: la gestión de la edad, http://www.insht.es/InshtWeb/Contenidos/Documentacion/FichasTecnicas/NTP/Fichero s/301a400/ntp_367.pdf (24/08/2017).

Martín López, S. (2006) Las empresas de participación ante el reto de la gestión del cambio demográfico. REVESCO. Revista de Estudios Cooperativos, Primer Cuatrimestre, $\mathrm{N}^{\circ}$ 88, pp. 99-127

Martínez Charterina, A. (2009) Innovación y Cooperativas. Boletín de la Asociación Internacional de Derecho Cooperativo, No 43, pp. 135-157.

Molina Navarrete, C. (2015) Envejecimiento activo versus prohibición de despido por edad ¿Quién debe pagar los costes laborales?. Estudios financieros. Revista de trabajo y seguridad social: Comentarios, casos prácticos: recursos humanos, $\mathrm{N}^{\mathrm{o}} 389-390$, pp. 123-129.

Molina Navarrete, C. (2017) Envejecimiento activo y jubilación: ¿Nuevo Retablo de las maravillas?. Estudios financieros. Revista de trabajo y seguridad social: Comentarios, casos prácticos: recursos humanos, № 412, pp. 5-16.

Monereo Pérez, J.L. y Fernández Bernat, J.A. (2016) Revalorización y actualización de pensiones: la STC 49/2015, de 5 de marzo de 2015. Revista de la Seguridad Social, ${ }^{\circ}$ Extraordinario 1, pp. 15-26.

MUTUA NAVARRA (2013) Gestión de la edad en la empresa. Reto y oportunidad. Pamplona: Ed. Colección Recomendaciones para una empresa saludable.

Ney, S. (2005) Active Ageing Policies in Europe: Between Path Dependency and Path Departure, Ageing International, No 4, Vol. 30, pp. 325-341.

Rey, P.M. (2012) Los trabajadores mayores y la estrategia Europa 2020. Revista Galega de Economía, vol.21, № Extraordinario (septiembre 2012), pp.1-23.

Ríos Mestre, J. (2017) La igualdad por razón de edad en el empleo. En: ADTSS. Balance y perspectivas de la política laboral y social europea. Madrid: Ed. Cinca, pp. 1-14. 
Sánchez Boza, R. (2012) Las empresas cooperativas ayudan a construir un mundo mejor. El caso centroamericano. Boletín de la Asociación Internacional de Derecho Cooperativo, $\mathrm{N}^{\mathrm{o}} 46$, pp. 33-52.

Sánchez-Urán Azaña, Y. (2011) Edad de jubilación y política de envejecimiento activo. Revista del Ministerio de Empleo y Seguridad Social, No 94, pp. 47-68.

Urrutikoetxea Barrutia, M. (2011) Envejecimiento y prevención de riesgos laborales. Lan Harremanak, $\mathrm{N}^{\circ} 24$, pp. 17-54.

Velázquez Fernández, M. (2015) El envejecimiento de la población trabajadora. Bilbao: Observatorio Vasco sobre acoso y discriminación. 